\title{
Psoralen promotes the expression of cyclin D1 in chondrocytes via the Wnt/ß-catenin signaling pathway
}

\author{
WENWEI ZHENG ${ }^{1 *}$, PINGDONG LIN ${ }^{1 *}$, YUHUAN MA ${ }^{1}$, XIANG SHAO ${ }^{2}$, HOUHUANG CHEN $^{2}$,

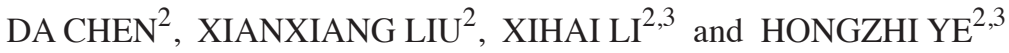 \\ ${ }^{1}$ College of Pharmacy, ${ }^{2}$ Academy of Integrative Medicine, ${ }^{3}$ Fujian Key Laboratory of Integrative Medicine on Geriatrics, \\ Fujian University of Traditional Chinese Medicine, Fuzhou, Fujian 350122, P.R. China
}

Received July 29, 2016; Accepted September 6, 2017

DOI: $10.3892 /$ ijmm.2017.3148

\begin{abstract}
Psoralen (PSO), the active ingredient of Fructus Psoraleae (FP) the dried ripe fruit of Psoralea corylifolia L., has been commonly used in traditional Chinese medicine (TCM) for the treatment of osteoarthritis (OA). We found that PSO activates cartilaginous cellular functions of rat chondrocytes in vitro. However, the effect of PSO on chondrocyte proliferation and the precise mechanisms involved remain to be elucidated. We investigated the effects of PSO on chondrocytes isolated from Sprague-Dawley (SD) rats and evaluated involvement of the Wnt $/ \beta$-catenin signaling pathway. The viability of chondrocytes treated with PSO was increased in a dose- and time-dependent manner, as assessed by MTT assay. We found that the gene expression and protein levels of Wnt-4, Frizzled-2, $\beta$-catenin and cyclin D1 in the PSO-treated chondrocytes were significantly upregulated, while the gene expression and protein level of glycogen synthase kinase-3 $\beta$ (GSK-3 $\beta$ ) were downregulated, compared with the untreated chondrocytes. By immunofluorescence, we also found that PSO induced $\beta$-catenin nuclear translocation. Importantly, the expression of $\beta$-catenin and cyclin D1 was partly inhibited by Dickkopf-1 (DKK-1), an inhibitor of the Wnt/ $\beta$-catenin signaling pathway. Additionally, Col-II expression in chondrocytes was increased after treatment with PSO. Taken together, these results indicate that PSO promotes chondrocyte proliferation by activating the Wnt/ $\beta$-catenin signaling pathway, and it may play an important role in the treatment of OA.
\end{abstract}

Correspondence to: Professor Xihai Li or Professor Hongzhi Ye, Academy of Integrative Medicine, Fujian University of Traditional Chinese Medicine, 1 Qiuyang Road, Minhou Shangjie, Fuzhou, Fujian 350122, P.R. China

E-mail: lixihai79dahai@163.com

E-mail: yelin0930@163.com

${ }^{*}$ Contributed equally

Key words: psoralen, Wnt/ $\beta$-catenin signaling pathway, chondrocytes, proliferation, osteoarthritis

\section{Introduction}

Osteoarthritis (OA), one of the most common chronic diseases of the joint and highly correlated with increasing age, is characterized by cartilage degeneration $(1,2)$. Cartilage is the dense connective tissue located in the joints between bones, and is composed of chondrocytes and extracellular matrix (ECM) (3). Cartilage degradation can lead to broken dynamic equilibrium between normal cartilage ECM synthesis and degradation. Chondrocytes, the only cell population of articular cartilage, are involved in maintaining cartilage homeostasis $(4,5)$. Cartilage degradation occurs in pathological conditions of $\mathrm{OA}$ (6). Therefore, improving and maintaining the proliferation potential and phenotype of chondrocytes are essential for the inhibition of cartilage degradation (7). This may potentially be an effective method with which to delay the development and progression of $\mathrm{OA}$.

The Wnt- $\beta /$ catenin signaling pathway is involved in the regulation of cartilage homeostasis, which plays a crucial role in the process of cell proliferation and the regulation of the chondrocyte phenotype $(8,9)$. Activation of the Wnt/ $\beta$-catenin signaling pathway is sufficient to induce dedifferentiation of articular chondrocytes (10). Following activation of the canonical Wnt/ $\beta$-catenin signaling pathway, $\beta$-catenin can accumulate in the cytoplasm and translocate to the nucleus, subsequently interacting with transcription factors such as T-cell factor and lymphoid enhancer binding factor (LEF) (11). Finally, these factors influence chondrocyte proliferation by affecting the expression of cyclin D1, a crucial factor in the cell cycle.

Psoralen (PSO) is one of the major active constituents of Fructus Psoraleae (Bu Gu Zhi) which is the dried ripe fruit of Psoralea corylifolia L. It has been commonly used in traditional Chinese medicine (TCM) for the treatment of osteoporosis, osteosarcoma, bone fracture, and osteomalacia (12). Studies have demonstrated that PSO stimulates local new bone formation in vivo and triggers osteogenesis $(13,14)$. A previous study showed that PSO may be used in the prevention and treatment of OA (15). However, the precise molecular mechanisms responsible for its effect on chondrocyte proliferation remain to be elucidated. Thus, our present study aimed to determine whether PSO promotes chondrocyte proliferation by activating the Wnt/ $\beta$-catenin signaling pathway. 


\section{Materials and methods}

Animals. Male Sprague-Dawley (SD) rats at 6 weeks of age were purchased from Shanghai SLAC Laboratory Animal Co., Ltd. (Shanghai, China). Animal experiments were strictly conducted in accordance with the Guide for the Care and Use of Laboratory Animals of Fujian University of Traditional Chinese Medicine (Fuzhou, China). The study protocol was approved by the Animal Care and Use Committee of Fujian University of Traditional Chinese Medicine. The SD rats were sacrificed using carbon dioxide (cage size, $7 \times 11 \times 5$ inches; flow rate, $1.3 \mathrm{l} / \mathrm{min}$ ), according to the Guide for the Care and Use of Animals.

Preparation of PSO-conditioned culture medium. PSO (psoralen, NIFDC, batch no. 110739-201115, 99.3\% pure) was first dissolved in phosphate-buffered-saline (PBS; HyClone Laboratories, Inc., Logan, UT, USA) to a concentration of $10^{-3} \mathrm{~mol} / \mathrm{l}$, and stored at $-20^{\circ} \mathrm{C}$. The PSO-conditioned culture medium was prepared by diluting the stock solution in low-Dulbecco's modified Eagle's medium (Low-DMEM) containing 10\% fetal bovine serum (FBS) (both from HyClone Laboratories, Inc.), filtering through a $0.22-\mu \mathrm{m}$ filter and stored at $4^{\circ} \mathrm{C}$ before use.

Isolation and identification of chondrocytes. Articular chondrocytes were isolated and cultured as previously described (16). The morphological changes and growth feature of chondrocytes were recorded under a phase-contrast microscope (Olympus, Tokyo, Japan). The primary chondrocytes were termed passage $0(\mathrm{P} 0)$; the $\mathrm{P} 2$ chondrocytes were identified by immunohistochemical staining of type II collagen. P2 chondrocytes approximately at 50\% were used in this study.

Cell viability analysis. Chondrocytes were seeded at the 96-well culture plates with a density of $2 \times 10^{4}$ cells $/ \mathrm{ml}$ and incubated $(100 \mu \mathrm{l} /$ well $)$ for $24 \mathrm{~h}$. The cells were then treated with PSO-conditioned culture medium at different concentrations $\left(0,10^{-8}, 10^{-7}, 10^{-6}, 10^{-5}\right.$ and $\left.10^{-4} \mathrm{~mol} / \mathrm{l}\right)$ for different periods $(24,48$ and $72 \mathrm{~h}$ ). At the end of intervention, the supernatant was then removed and $100 \mu 1$ 1\% MTT (Sigma-Aldrich) was added to each well.After $4 \mathrm{~h}$ of incubation at $37^{\circ} \mathrm{C}$, the supernatant was replaced with $150 \mu \mathrm{l} /$ well of DMSO (Hengxing Chemical Preparation Co., Ltd., Tianjin, China). The absorbance was measured at $490 \mathrm{~nm}$ using an enzyme labeling instrument (model EXL800; BioTek, Winooski, VT, USA). The optimum conditions of intervention were selected for further examination. To further investigate whether PSO promotes chondrocyte proliferation via the canonical Wnt- $\beta /$ catenin signaling pathway, the chondrocytes were treated with PSO $\left(10^{-6} \mathrm{~mol} / \mathrm{l}\right)$ in the absence or presence of $0.2 \mu \mathrm{g} / \mathrm{ml}$ Dickkopf-1 (DKK-1; R\&D Systems, Minneapolis, MN, USA) for $48 \mathrm{~h}$, as previously described (17).

RNA extraction and RT-PCR analysis. After treatment, total RNA was extracted from the cells using TRIzol reagent (Invitrogen, Grand Island, NY, USA). RNA $(1 \mu \mathrm{g})$ was reverse transcribed into cDNA using a reverse transcription kit (Thermo Fisher Scientific, Inc., Rockford, IL, USA) according to the manufacturer's instructions. Then DNA bands were analyzed via gel electrophoresis (1.5\% agarose) using the Gel Documentation System (Bio-Rad, Hercules, CA, USA) and normalized to that of $\beta$-actin. The PCR primers were as follows: $\beta$-actin forward, 5'-GAG AGG GAA ATC GTG CGT GAC-3' and reverse, 5'-CAT CTG CTG GAA GGT GGA CA-3'; Wnt-4 forward, 5'-TCA GCC CAC AGG GTT TCC A-3' and reverse, 5'-CGC TCG CCA GCA TGT CTT T-3'; $\beta$-catenin forward, 5'-AAG GAA GCT TCC AGA CAT GC-3' and reverse, 5'-AGC TTG CTC TCT TGA TTG CC-3'; Frizzled-2 forward, 5'-TCG AGG CCA ATT CGC AGT A-3' and reverse, 5'-CAG GAA GGA TGT GCC GAT G-3'; glycogen synthase kinase-3 $\beta$ (GSK-3 $\beta$ ) forward, 5'-AAA GTG CAT CGC TGG CTT A-3' and reverse, 5'-GTC GAC GGT TTG TTT CCA AT-3'; cyclin D1 forward, 5'-AAT GCC AGA GGC GGA TGA GA-3' and reverse, 5'-GCT TGT GCG GTA GCA GGA GA-3'; Col-II forward, 5'-CCA GAG TGG AAG AGC GGA GAC-3' and reverse, 5'-CAG TGG ACA GTA GAC GGA GGA AAG-3'; and $\beta$-actin forward, 5'-CAC CCG CGA GTA CAA CCT TC-3' and reverse, 5'-CCC ATA CCC ACC ATC ACA CC-3'.

Western blot analysis. After treatment, total proteins were extracted from cells using radioimmunoprecipitation assay lysis buffer (RIPA) with $1 \mathrm{mM}$ phenylmethanesulfony fluoride (PMSF) (both from Beyotime Biotechnology, Shanghai, China) and quantified using a bicinchoninic acid (BCA) assay. Twenty micrograms of proteins were separated on sodium dodecyl sulfate-polyacrylamide gel electrophoresis (SDS-PAGE) gels (12\%) and transferred onto a PVDF membrane. Subsequently, the membrane was blocked with 5\% non-fat milk in TBST solution for $2 \mathrm{~h}$ at room temperature (RT). After incubation with the primary antibodies against Wnt-4 (sc-5214), Frizzled-2 (sc-68327; Santa Cruz Biotechnology, Inc., Santa Cruz, CA, USA), $\beta$-catenin (9582s), GSK-3 $\beta$ (9315; Cell Signaling Technology, Inc., Beverly, MA, USA), cyclin D1 (BS6532), Col-II (BS1071; Bioworld Technology, Natong, China) overnight at $4^{\circ} \mathrm{C}$, and the HRP-conjugated secondary antibodies (ZB-2301; Zhongshan Golden Bridge Biotechnology Co., Ltd.) for $1 \mathrm{~h}$ at RT, the blots were detected using a Bio-Rad Chemi Doc XRS+ (Bio-Rad), and $\beta$-actin was used as the control.

Immunofluorescence staining. After treatment with PSO at the concentration of $10^{-6} \mathrm{~mol} / \mathrm{l}$, chondrocytes were fixed with ice-cold methanol at $4^{\circ} \mathrm{C}$ for $30 \mathrm{~min}$, permeabilized with $0.5 \%$ Triton X-100 for 10 min, and blocked in $5 \%$ bovine serum albumin (BSA) for $1 \mathrm{~h}$ at room temperature. Then the cells were incubated with rabbit anti- $\beta$-catenin antibody overnight at $4^{\circ} \mathrm{C}$, and TRITC-conjugated secondary antibody (Zymed Laboratories, San Francisco, CA, USA) was applied for $1 \mathrm{~h}$ at room temperature in the dark. Following DAPI staining, confocal images were acquired with a fluorescence microscope (Olympus, Tokyo, Japan).

Statistical analysis. All the experiments were repeated three times independently and all the data were analyzed by one way analysis of variance (ANOVA) or Student's t-test using SPSS 19.0 software (SPSS Inc, Chicago, IL, USA). $\mathrm{P}<0.05$ was considered to indicate a statistically significant difference. 
A

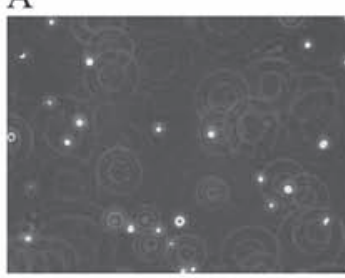

B

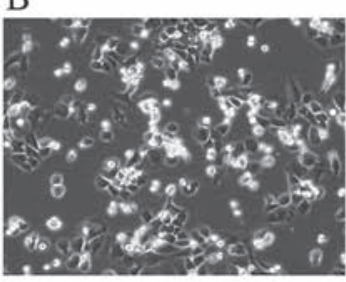

C

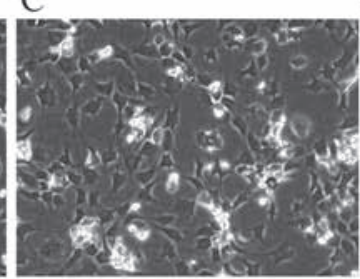

D

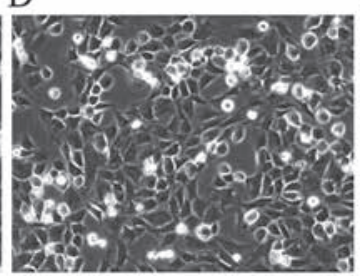

E

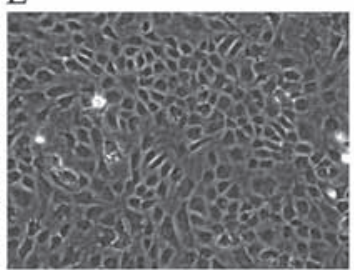

Figure 1. Morphology of chondrocytes (magnification, x200). (A) Newly isolated chondrocytes. Primary cells cultured for (B) 24 h, (C) 4 days and (D) 8 days. (E) P2 chondrocytes cultured for 4 day.

A

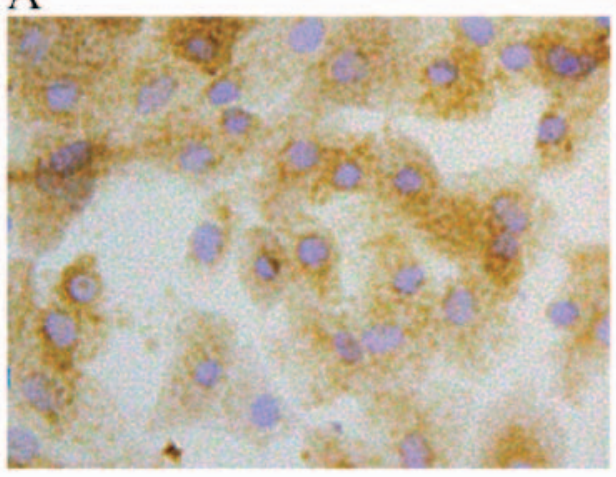

B

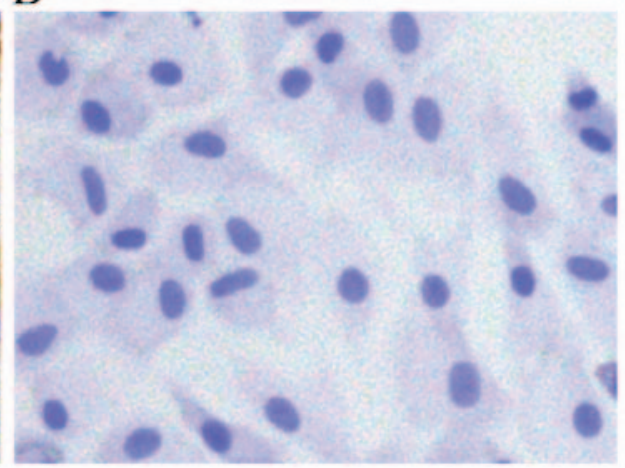

Figure 2. Identification of chondrocytes by type II collagen immunohistochemistry (magnification, x200). (A) Positive chondrocytes were stained brown in the cytoplasm. (B) Negative chondrocytes negative for type II collagen were not stained.
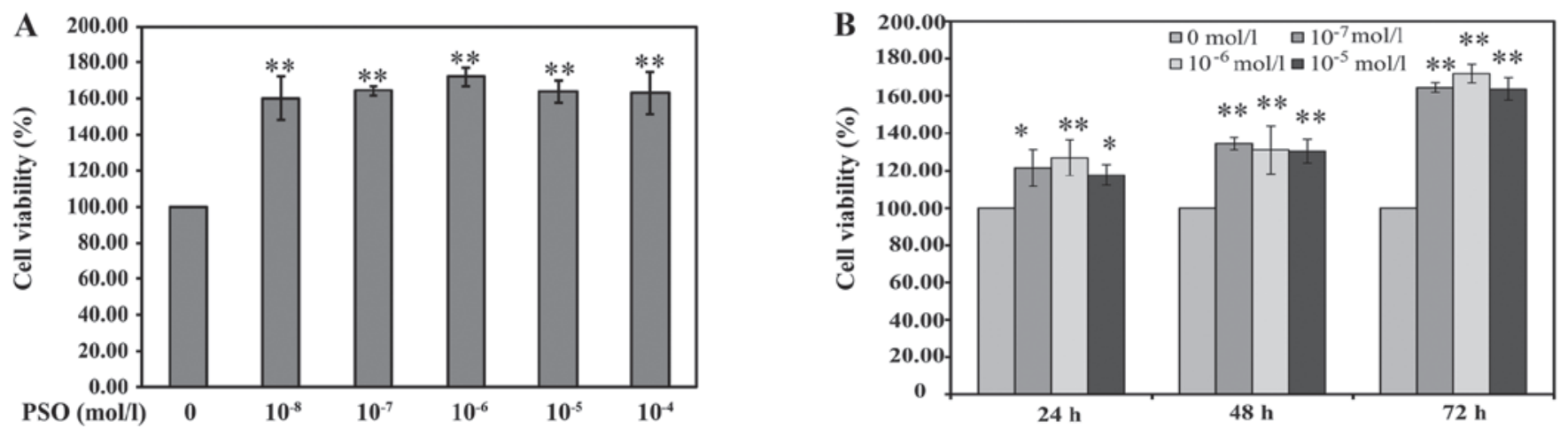

Figure 3. Psoralen (PSO) enhances the cell viability of chondrocytes. (A) Chondrocytes treated with different concentrations of PSO for 72 h. (B) Chondrocytes treated with $10^{-7}, 10^{-6}, 10^{-5} \mathrm{~mol} / 1$ PSO for different times. ${ }^{*} \mathrm{P}<0.05$ and ${ }^{* *} \mathrm{P}<0.01$ compared with the untreated chondrocytes (treated with 0 mol/1 PSO).

\section{Results}

Morphology and identification of chondrocytes. The cultured chondrocytes in this study exhibited typical morphology with a spherical, fusiform and slab stone shape, as described in previous studies $(17,18)$ (Fig. 1). The P2 chondrocytes exhibited a typical polygonal or spherical shape, as shown by the identification of type II collagen immunohistochemical staining. The cytoplasm of positive chondrocytes was stained brown, whereas the negative control failed to stain in the cytoplasm (Fig. 2).

PSO increases chondrocyte viability. The effect of PSO on cell viability was measured by the MTT assay. As shown by the results of the MTT assay, the viability of the chondrocytes was increased following treatment with different PSO dosages of $10^{-8}-10^{-4} \mathrm{~mol} / \mathrm{l}$ and for increasing time periods as compared with the viability noted in the untreated cells. The cell viability of the $10^{-6} \mathrm{~mol} / \mathrm{l} \mathrm{PSO}$ concentration treatment group was slightly higher than that in the other PSO concentration treatment groups. However, there was no significant difference among the $10^{-8}-10^{-4} \mathrm{~mol} / 1 \mathrm{PSO}$ concentration treatment groups. Furthermore, treatments with $10^{-7}, 10^{-6}$ and $10^{-5} \mathrm{~mol} / \mathrm{l}$ of PSO were more effective on the third day compared with the first day, and all the comparisons showed significant difference $(\mathrm{P}<0.01$ or $\mathrm{P}<0.05)$ (Fig. 3$)$. These results preliminarily indicate that PSO promotes the viability of chondrocytes in a time- and concentration-dependent manner. Thus, the 
A

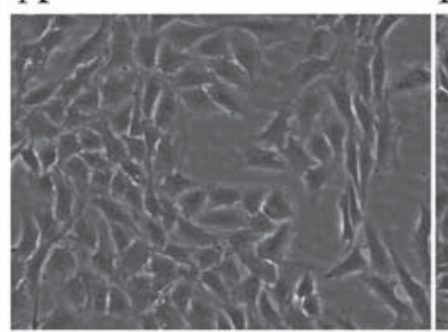

PSO (mol/l)
$\mathrm{B}$

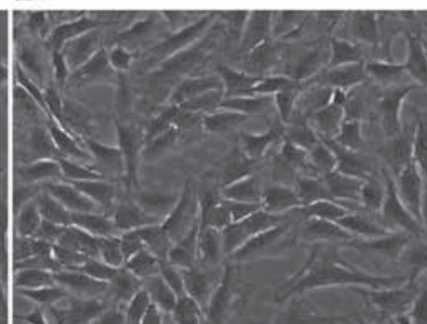

$10^{-7}$
$\mathrm{C}$

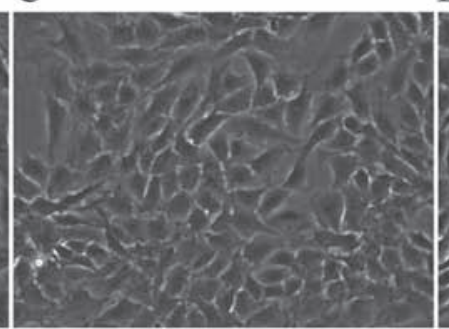

$10^{-6}$
$\mathrm{D}$

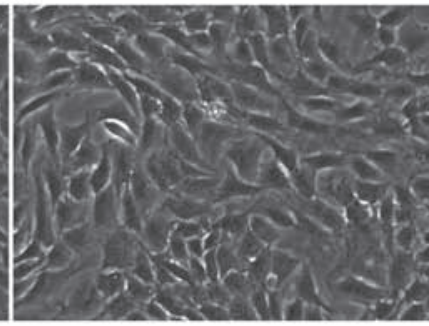

$10^{-5}$

Figure 4. Morphological change in chondrocytes treated with different concentrations of psoralen (PSO) for $48 \mathrm{~h}$ (magnification, x200). (A) Untreated cells. (B) Chondrocytes treated with $10^{-7} \mathrm{~mol} / 1 \mathrm{PSO}$. (C) Chondrocytes treated with $10^{-6} \mathrm{~mol} / 1 \mathrm{PSO}$. (D) Chondrocytes treated with $10^{-5} \mathrm{~mol} / 1 \mathrm{PSO}$.

A
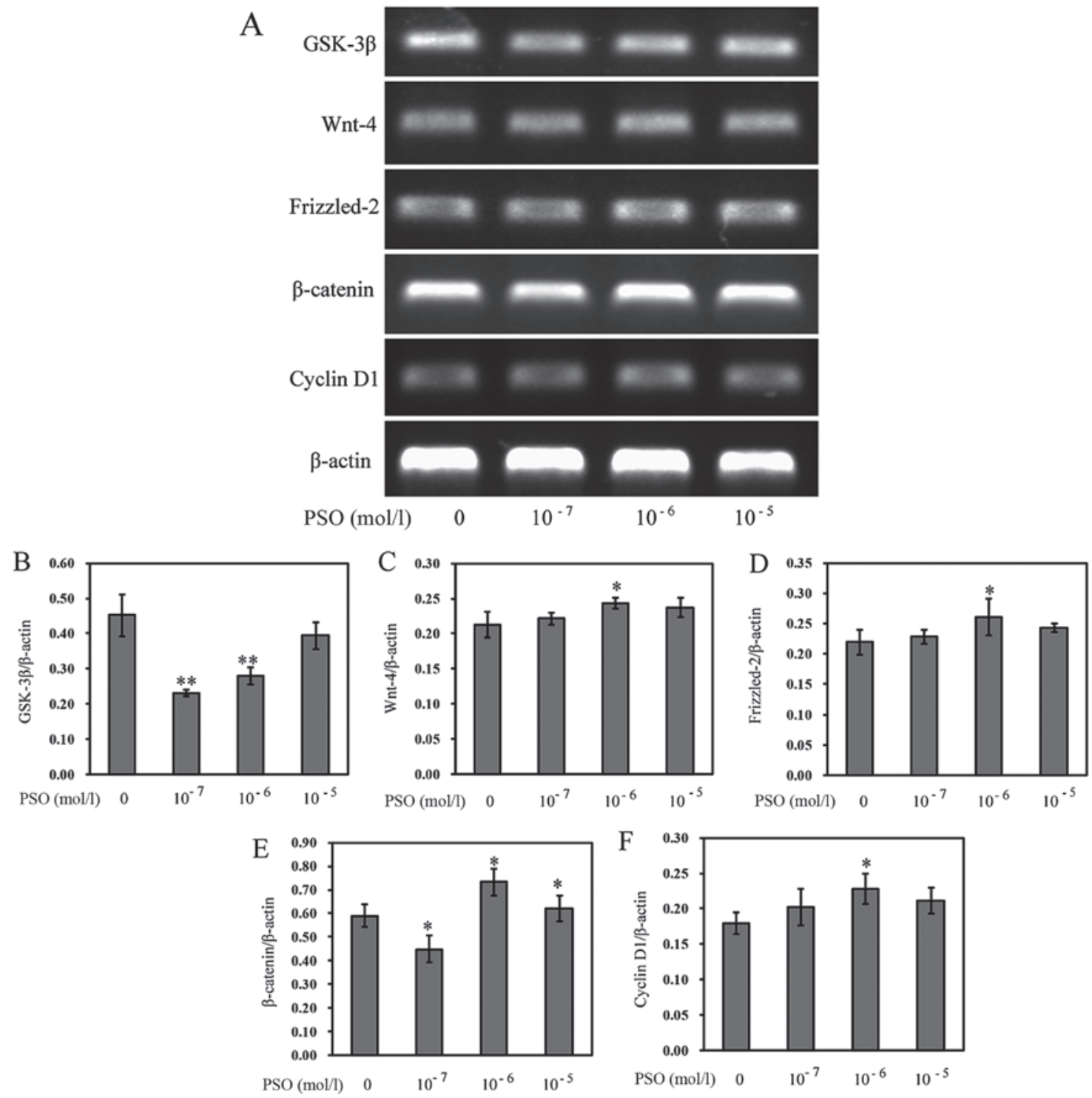

Figure 5. Psoralen (PSO) increases the mRNA expression of Wnt-4, Frizzled-2, $\beta$-catenin, cyclin D1, and decreases the expression of glycogen synthase

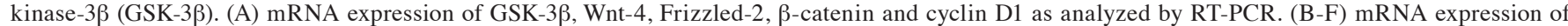
(B) GSK-3 $\beta$, (C) Wnt-4, (D) Frizzled-2, (E) $\beta$-catenin, and (F) cyclin D1 in chondrocytes treated with or without PSO. "P<0.05 and ${ }^{* *} \mathrm{P}<0.01$ compared with the untreated chondrocytes (treated with $0 \mathrm{~mol} / \mathrm{l} \mathrm{PSO}$ ).

concentrations of $10^{-7}, 10^{-6}$ and $10^{-5} \mathrm{~mol} / 1$ (low, medium and high doses, respectively) of PSO for a 48-h incubation were used for further examination.
The morphology of the PSO-treated chondrocytes at $48 \mathrm{~h}$ was observed by phase-contrast microscope. Compared with the untreated cells, the PSO-treated chondrocytes exhibited 
A
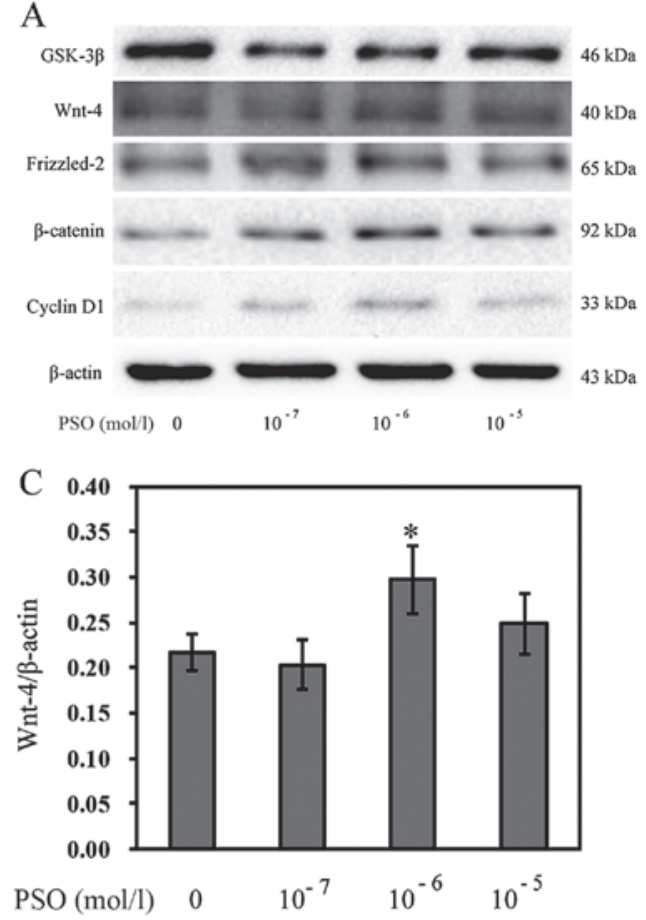

$\mathrm{E}$

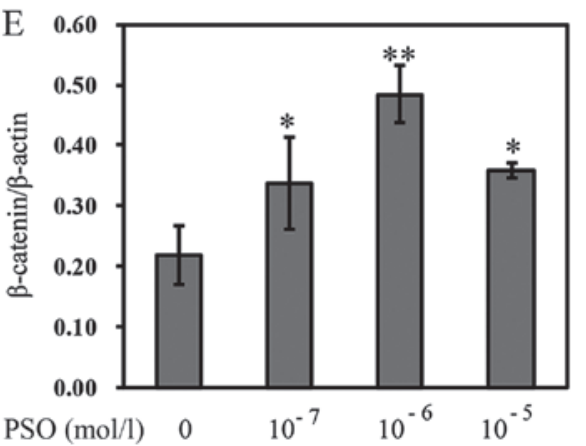

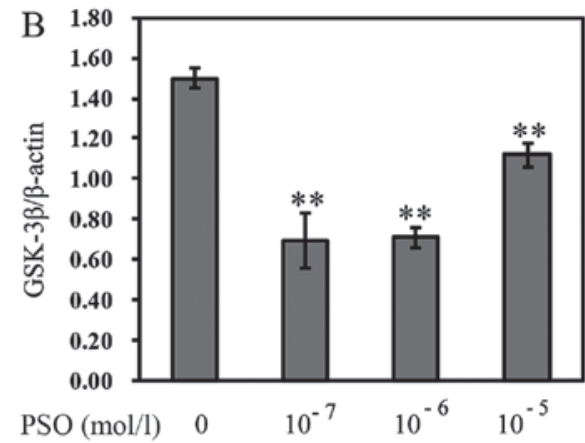
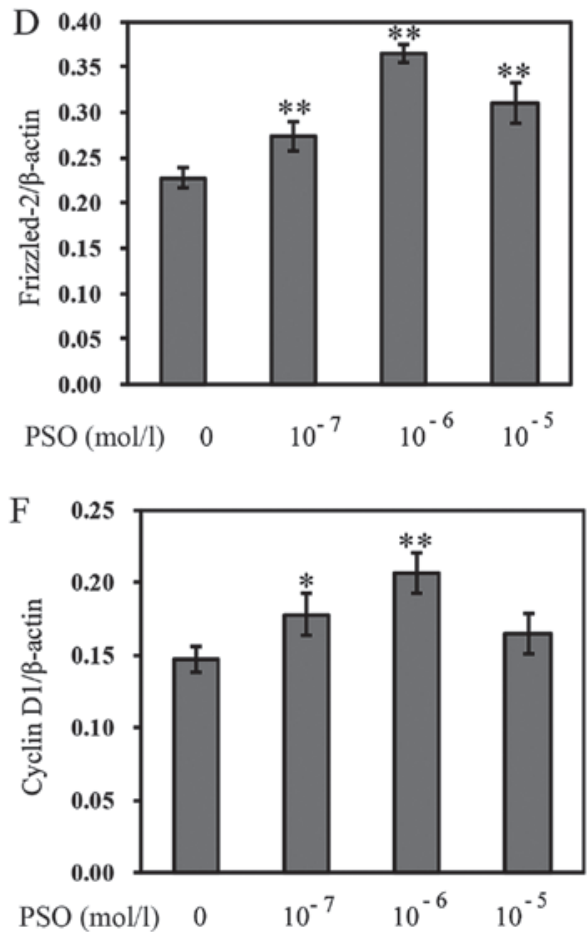

Figure 6. Psoralen (PSO) increases the protein levels of Wnt-4, Frizzled-2, $\beta$-catenin, cyclin D1, and decreases the protein level of glycogen synthase kinase-3 $\beta$ (GSK-3 $\beta$ ). (A) The protein levels of GSK-3 $\beta$, Wnt-4, Frizzled-2, $\beta$-catenin and cyclin D1 as analyzed by western blotting. (B-E) The protein levels of (B) GSK-3 $\beta$, (C) Wnt-4, (D) Frizzled-2, (E) $\beta$-catenin and (F) cyclin D1 in chondrocytes treated with or without PSO. ${ }^{*}<0.05$ and ${ }^{* *} \mathrm{P}<0.01$ compared with the untreated chondrocytes (treated with $0 \mathrm{~mol} / \mathrm{l} \mathrm{PSO}$ ).

changes in cell size and shape, and in particular an increase in cell number (Fig. 4).

PSO upregulates the expression of Wnt-4, Frizzled-2, $\beta$-catenin, cyclin D1 and downregulates the expression of $G S K-3 \beta$. To evaluate the effect of PSO on the Wnt/ $\beta$-catenin signaling pathway in chondrocytes, RT-PCR and western blot analysis were performed to determine the expression levels of Wnt-4, Frizzled-2, $\beta$-catenin, cyclin D1 and GSK-3 $\beta$ in chondrocytes. Compared with the control group, PSO efficiently upregulated the mRNA expression of Wnt-4, Frizzled-2, $\beta$-catenin, cyclin D1 $(\mathrm{P}<0.01$ or $\mathrm{P}<0.05)$, but downregulated the mRNA expression of GSK-3 $\beta(\mathrm{P}<0.01$ or $\mathrm{P}<0.05)$ (Fig. 5). Similarly, the protein levels, respectively, corresponded to the mRNA expression $(\mathrm{P}<0.01$ or $\mathrm{P}<0.05)$ (Fig. 6).

PSO promotes $\beta$-catenin nuclear translocation. To gain insight into the effect of PSO on the promotion of $\beta$-catenin nuclear translocation, immunofluorescence staining was applied. According to the staining results, we found that PSO mark- edly promoted the translocation of $\beta$-catenin into the nucleus $(\mathrm{P}<0.01$ or $\mathrm{P}<0.05)$ (Fig. 7$). \beta$-catenin is a protein that mainly localizes in the membrane with minimal localization in the cytoplasm. It can enter into the nucleus to transactivate target gene expression upon activation in the cytoplasm (19). These results indicate that PSO activates the $\mathrm{Wnt} / \beta$-catenin signaling pathway by promoting $\beta$-catenin nuclear translocation.

PSO increases the expression of Col-II. The loss of type II collagen is a characteristic of articular cartilage degradation. Thus, we investigated the influence of PSO on Col-II expression. The results revealed that the mRNA expression and protein level of Col-II were markedly enhanced in the PSO-treated chondrocytes $(\mathrm{P}<0.01$ or $\mathrm{P}<0.05)$ (Fig. 8), compared with levels in the untreated cells, indicating that PSO upregulates the expression of Col-II to promote cartilage ECM synthesis, and confer a positive effect on chondrocyte proliferation.

Expression of $\beta$-catenin and cyclin D1 in the Wnt/ $\beta$-catenin signaling pathway is inhibited by $D K K-1$. To further confirm 

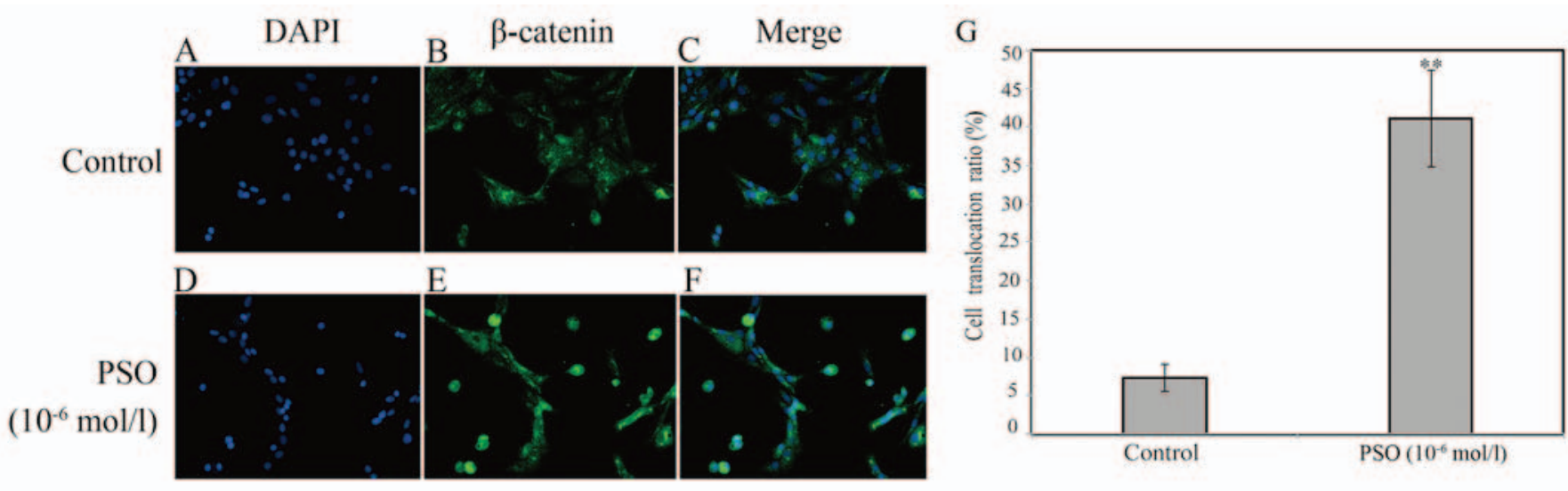

Figure 7. Psoralen (PSO) enhances $\beta$-catenin nuclear translocation in chondrocytes. Chondrocytes were treated with $10^{-6} \mathrm{~mol} / 1 \mathrm{PSO}$ for $48 \mathrm{~h}$, followed by immunofluorescence staining for $\beta$-catenin (green) and the nuclei were stained with DAPI (blue). (A-F) The immunofluorescence staining revealed that $\beta$-catenin was enriched in the nuclei of PSO-treated chondrocytes, while it was mainly localized in the cytoplasm and on the cell membrane in normal chondrocytes. (G) Quantitative analysis confirmed the cell translocation ratio $(\%)$. Cell translocation ratio $(\%)=($ number of cells enriched in $\beta$-catenin in the nuclei/number of total cells) $\mathrm{x} 100 .{ }^{* *} \mathrm{P}<0.01$ compared to untreated chondrocytes (treated with $0 \mathrm{~mol} / \mathrm{PSO}$ ).
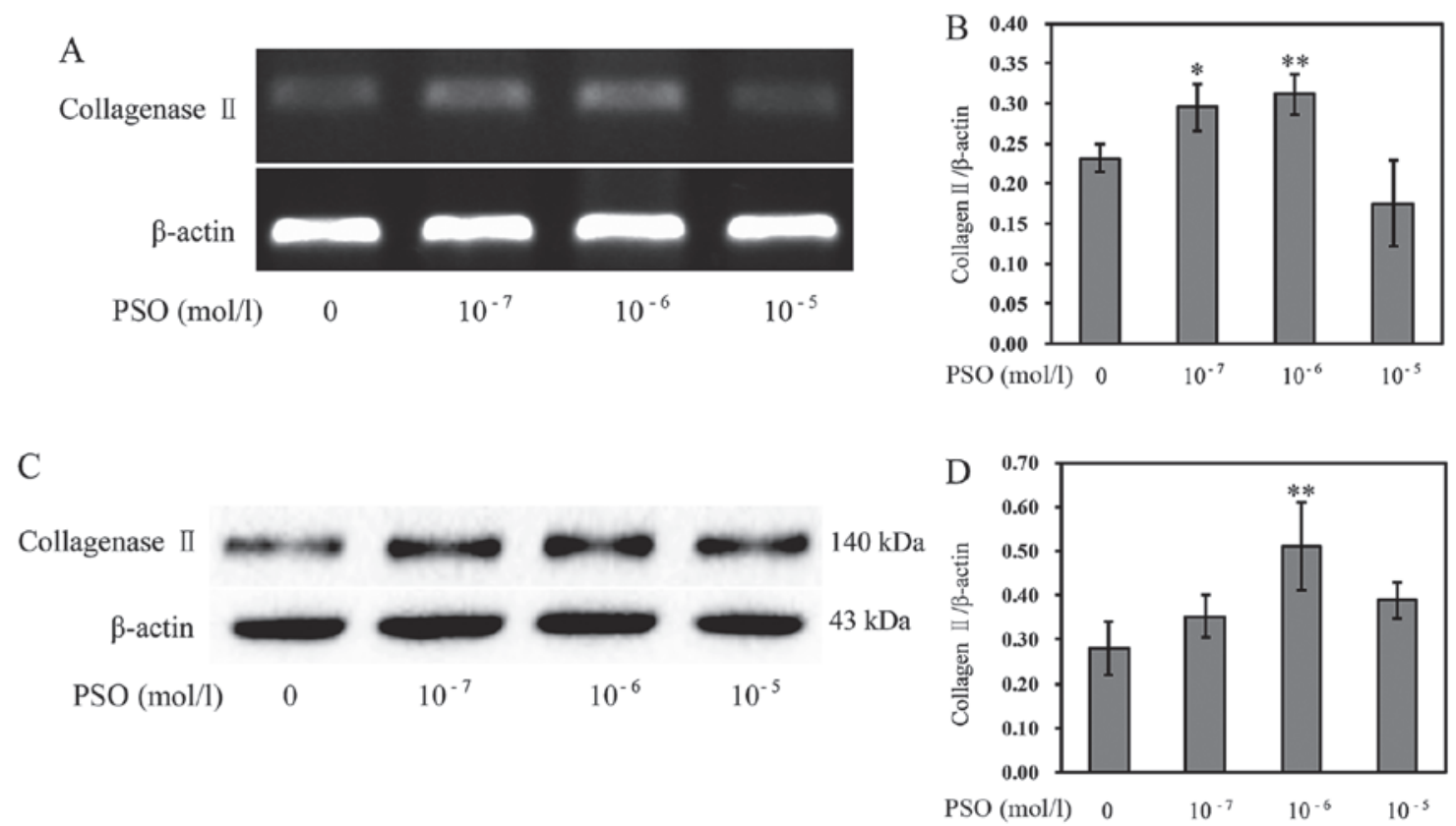

Figure 8. Psoralen (PSO) increases the expression of type II collagen. (A and B) mRNA expression of type II collagen. (C and D) The protein level of type II collagen. ${ }^{*} \mathrm{P}<0.05$ and ${ }^{* *} \mathrm{P}<0.01$ compared with the untreated chondrocytes (treated with $0 \mathrm{~mol} / 1 \mathrm{PSO}$ ).

the effects of PSO on chondrocyte proliferation by activating the Wnt/ $\beta$-catenin signaling pathway, we also investigated the influences of $\mathrm{Wnt} / \beta$-catenin signaling inhibition on $\beta$-catenin and cyclin D1 expression. The expression of $\beta$-catenin and cyclin D1 was decreased in cells pre-treated with DKK-1 compared with that without DKK-1 treatment $(\mathrm{P}<0.01$ or $\mathrm{P}<0.05$ ), implying that PSO is involved in the regulation of chondrocyte proliferation by activating the $\mathrm{Wnt} / \beta$-catenin signaling pathway (Fig. 9).

\section{Discussion}

$\mathrm{OA}$ is a progressively degenerative joint disorder that is gradually becoming a major health issue among the aged population worldwide (20). Chondrocytes are responsible for the production and maintenance of the ECM. In OA, dysfunction of articular chondrocytes causes degradation of the ECM exceeding its synthesis, leading to the degradation of cartilage (21). Thus, the development of effective agents for the protection of chondrocytes to treat $\mathrm{OA}$ is crucial. However, currently no effective medical therapy is supported for OA except physiotherapy and surgery. In the present study, we found that PSO enhanced chondrocyte viability in a doseand time-dependent manner as determined by MTT assay, indicating that PSO is a potential therapeutic agent for the treatment of OA. Thus, we further investigated the mechanisms involved in the chondrocyte proliferation mediated by PSO. 


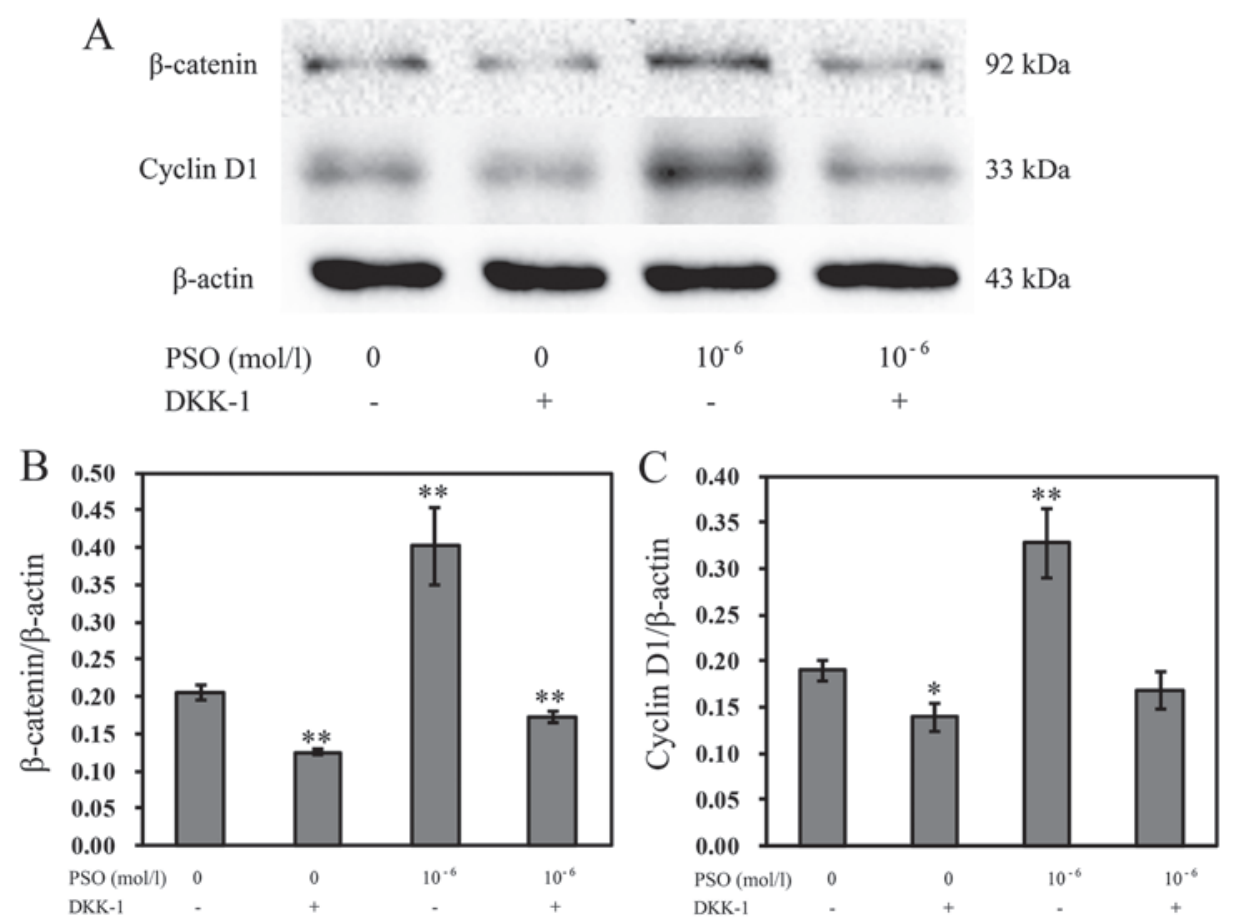

Figure 9. Effect of Dickkopf-1 (DKK-1) on the psoralen (PSO)-induced expression of cyclin D1 and $\beta$-catenin. Chondrocytes were incubated with PSO $\left(10^{-6} \mathrm{~mol} / \mathrm{l}\right)$ in the absence or presence of $0.2 \mu \mathrm{g} / \mathrm{ml}$ DKK-1. (A) The protein levels of $\beta$-catenin and cyclin D1 were assessed. (B and C) The protein levels of (B) $\beta$-catenin and (C) cyclin D1. $\mathrm{P}<0.05$ and ${ }^{* * *} \mathrm{P}<0.01$ compared with the untreated chondrocytes (treated with $0 \mathrm{~mol} / \mathrm{l} \mathrm{PSO}$ ).

The Wnt/ $\beta$-catenin signaling pathway plays a vital role in a number of cellular events including cell proliferation, migration and differentiation. The $\mathrm{Wnt} / \mathrm{\beta}$-catenin signaling pathway is important in the regulation of proliferation. It regulates chondrogenes at different stages in different ways, and plays a crucial role in the physiopathology of OA (22-24). Altering the expression of genes and proteins in articular chondrocytes is correlated with the pathological process of OA (25). Several molecular components of the Wnt/ $\beta$-catenin signaling pathway have been shown to be associated with OA, including Wnt- 4 , Frizzled-2, $\beta$-catenin and GSK-3 $\beta$. The Wnt $/ \beta$-catenin signaling pathway is triggered by binding of Wnt ligands to frizzled receptors and co-receptors, low-density lipoprotein receptors (LRP)5/6. GSK-3 phosphorylates LRP5/6 which results in recruitment of the axin complex to this co-receptor and leads to reduced degradation of $\beta$-catenin. Next, the resultant accumulated cytoplasmic $\beta$-catenin translocates to the nucleus $(26,27)$. Finally, this activates target genes, such as cyclin D1, a positive effector of the G1/S transition that is particularly involved in cell proliferation $(11,28)$. In the present study, we investigated the activity of PSO in the promotion of chondrocyte proliferation. Our results showed that the expression of Wnt-4, Frizzled-2, $\beta$-catenin and cyclin D1 in PSO-treated chondrocytes was markedly increased, whereas the expression of GSK-3 $\beta$ was significantly decreased. Additionally, PSO contributed to increased $\beta$-catenin nuclear translocation in the chondrocytes. These results revealed that PSO activates the Wnt/ $\beta$-catenin signaling pathway to promote chondrocyte proliferation.

DKK-1, an inhibitor of the $\mathrm{Wnt} / \beta$-catenin signaling pathway, antagonizes WNT signaling by interacting with co-receptor LRP leading to LRP endocytosis (29). DKK-1 inhibits the Wnt signaling pathway by binding to LRP5/6 on target cells. Following the inhibition, GSK-3 directly phosphorylates the transcriptional regulator $\beta$-catenin, marking it for proteosomal degradation, and then partly decreasing the expression of $\beta$-catenin and cyclin D1 (30). Our results revealed that the expression of $\beta$-catenin and cyclin D1 was partly inhibited by DKK-1, which further demonstrated that PSO promoted chondrocyte proliferation via the Wnt/ $\beta$ catenin signaling pathway.

In conclusion, the present study demonstrated that PSO promoted chondrocyte proliferation by regulating the Wnt/ $\beta$-catenin signaling pathway. Further studies using animal models should be carried out to investigate the detailed mechanisms of the Wnt $/ \beta$-catenin signaling pathway in the pathogenesis of OA. In addition, the present study also found that PSO increased the expression of Col-II in chondrocytes, while this was partly inhibited by DKK-1. This result indicated that PSO has a positive effect on preventing cartilage degradation by increasing the expression of Col-II, a major component of the cartilage matrix. Future studies using animal models should investigate the detailed mechanism of the Wnt/ $\beta$-catenin signaling pathway in the pathogenesis of OA.

\section{Acknowledgements}

The present study was supported by the National Natural Science Foundation of China (grant no. 81373818), the Key Project of Fujian Provincial Department of Science and Technology Department (grant no. 2014Y0064), the Natural Science Foundation of Fujian Province (grant nos. 2014J01357 and 2016J01395) and the Developmental Fund of Chen Keji Integrative Medicine (grant no. CKJ2015009). 


\section{References}

1. Pereira D, Ramos E and Branco J: Osteoarthritis. Osteoarthritis Acta Med Port 28: 99-106, 2015.

2. Blalock D, Miller A, Tilley M and Wang J: Joint instability and osteoarthritis. Clin Med Insights Arthritis Musculoskelet Disord 8: 15-23, 2015.

3. Thomas JT and Schneider BS: Frank EL andKrizan SJ: Cartilage repair and replacement in the knee. Trends Biotechnol 31: 665-667, 2013

4. Chen C, Tambe DT, Deng L and Yang L: Biomechanical properties and mechanobiology of the articular chondrocyte. Am J Physiol Cell Physiol 305: C1202-C1208, 2013.

5. Tonge DP, Pearson MJ and Jones SW: The hallmarks of osteoarthritis and the potential to develop personalised disease-modifying pharmacological therapeutics. Osteoarthritis Cartilage 22 : 609-621, 2014

6. Schulze-Tanzil G: Activation and dedifferentiation of chondrocytes Implications in cartilage injury and repair. Ann Anat 191: 325-338, 2009.

7. Pap T and Korb-Pap A: Cartilage damage in osteoarthritis and rheumatoid arthritis - two unequal siblings. Nat Rev Rheumatol 11 606-615, 2015

8. Aman A, Nguyen M and Piotrowski T: Wnt/ $\beta$-catenin dependent cell proliferation underlies segmented lateral line morphogenesis. Dev Biol 349: 470-482, 2011.

9. Monroe DG, McGee-Lawrence ME, Oursler MJ and Westendorf JJ: Update on Wnt signaling in bone cell biology and bone disease. Gene 492: 1-18, 2012.

10. Ryu JH, Kim SJ, Kim SH, Oh CD, Hwang SG, Chun CH, Oh SH, Seong JK, Huh TL and Chun JS: Regulation of the chondrocyte phenotype by beta-catenin. Development 129: 5541-5550, 2002.

11. Bougault C, Priam S, Houard X, Pigenet A, Sudre L, Lories RJ Jacques $\mathrm{C}$ and Berenbaum F: Protective role of frizzled-related protein $\mathrm{B}$ on matrix metalloproteinase induction in mouse chondrocytes. Arthritis Res Ther 16: R137, 2014.

12. Lu H, Zhang L, Liu D, Tang $P$ and Song F: Isolation and purification of psoralen and isopsoralen and their efficacy and safety in the treatment of osteosarcoma in nude rats. Afr Health Sci 14: 641-647, 2014.

13. Wong RW and Rabie AB: Effect of psoralen on bone formation. J Orthop Res 29: 158-164, 2011.

14. Tang DZ, Yang F, Yang Z, Huang J, Shi Q, Chen D and Wang YJ: Psoralen stimulates osteoblast differentiation through activation of BMP signaling. Biochem Biophys Res Commun 405: 256-261, 2011.

15. Li J, Zhu A, Xie W and Zheng H: Study of the mechanism of benefiting kidney herbs in the prevention and treatment of osteoarthritis in vitro. Chin J Osteoporos 22: 877-882, 2016 (In Chinese).

16. 16. Li H, Li X, Liu G, Chen J, Weng X, Liu F, Xu H, Liu X and Ye H: Bauhinia championi (Benth.) Benth. polysaccharides upregulate Wnt/ $\beta$-catenin signaling in chondrocytes. Int $\mathrm{J}$ Mol Med 32: 1329-1336, 2013.
17. Weng X, Lin P, Liu F, Chen J, Li H, Huang L, Zhen C, Xu H, Liu X, Ye H, et al: Achyranthes bidentata polysaccharides activate the Wnt/ $\beta$-catenin signaling pathway to promote chondrocyte proliferation. Int J Mol Med 34: 1045-1050, 2014.

18. Yu F, Li X, Cai L, Li H, Chen J, Wong X, Xu H, Zheng C, Liu X and Ye $\mathrm{H}$ : Achyranthes bidentata polysaccharides induce chondrocyte proliferation via the promotion of the G1/S cell cycle transition. Mol Med Rep 7: 935-940, 2013.

19. Maturana JL, Niechi I, Silva E, Huerta H, Cataldo R, Härtel S, Barros LF, Galindo M and Tapia JC: Transactivation activity and nucleocytoplasmic transport of $\beta$-catenin are independently regulated by its C-terminal end. Gene 573: 115-122, 2015.

20. Hunter DJ and Felson DT: Osteoarthritis. BMJ 332: 639-642, 2006.

21. Dreier R: Hypertrophic differentiation of chondrocytes in osteoarthritis: The developmental aspect of degenerative joint disorders. Arthritis Res Ther 12: 216, 2010.

22. Tao HY, He B, Liu SQ, Wei AL, Tao FH, Tao HL, Deng WX, Li HH and Chen Q: Effect of carboxymethylated chitosan on the biosynthesis of NGF and activation of the Wnt/ $\beta$-catenin signaling pathway in the proliferation of Schwann cells. Eur J Pharmacol 702: 85-92, 2013.

23. Sassi N, Laadhar L, Allouche M, Achek A, Kallel-Sellami M, Makni S and Sellami S: WNT signaling and chondrocytes: From cell fate determination to osteoarthritis physiopathology. J Recept Signal Transduct Res 34: 73-80, 2014.

24. Sassi N,Laadhar L, Allouche M,Zandieh-Doulabi B,Hamdoun M, Klein-Nulend J, Makni S and Sellami S: The roles of canonical and non-canonical Wnt signaling in human de-differentiated articular chondrocytes. Biotech Histochem 89: 53-65, 2014.

25. Li X, Chen J, Liang W, Li H, Liu F, Weng X, Lin P, Chen W, Zheng $\mathrm{C}, \mathrm{Xu} \mathrm{H}$, et al: Bushen Zhuangjin Decoction promotes chondrocyte proliferation by stimulating cell cycle progression. Exp Ther Med 9: 839-844, 2015.

26. Zeng X, Tamai K, Doble B, Li S, Huang H, Habas R, Okamura H, Woodgett $\mathrm{J}$ and $\mathrm{He} \mathrm{X}$ : A dual-kinase mechanism for Wnt co-receptor phosphorylation and activation. Nature 438: 873-877, 2005.

27. Doble BW, Patel S, Wood GA, Kockeritz LK and Woodgett JR Functional redundancy of GSK-3alpha and GSK-3beta in Wnt/beta-catenin signaling shown by using an allelic series of embryonic stem cell lines. Dev Cell 12: 957-971, 2007.

28. MacDonald BT, Tamai $\mathrm{K}$ and $\mathrm{He} \mathrm{X}$ : Wnt/ $\beta$-catenin signaling: Components, mechanisms, and diseases. Dev Cell 17: 9-26, 2009

29. Mao B and Niehrs C: Kremen 2 modulates Dickkopf 2 activity during Wnt/LRP6 signaling. Gene 302: 179-183, 2003.

30. Liu C, Li Y, Semenov M, Han C, Baeg GH, Tan Y, Zhang Z, Lin $\mathrm{X}$ and He $\mathrm{X}$ : Control of beta-catenin phosphorylation/degradation by a dual-kinase mechanism. Cell 108: 837-847, 2002. 